\title{
Saving lives and protecting people from injuries and violence
}

\section{Debra Houry}

Injury is the leading cause of death for people aged 1-44 in the USA. CDC's National Center for Injury Prevention and Control—-the Injury Center-is uniquely poised to measure the toll of injury and violence on the lives of Americans and reduce factors that increase their risk. For more than 20 years, Injury Center scientists and partners in the field have tracked trends in injury and violence burden, investigated risk factors through surveillance and research and translated findings into evidence-based strategies and interventions.

The Injury Center has two CDC agency-wide priorities: (1) motor vehicle injury and (2) prescription drug overdose, and has identified the topics of child abuse and neglect, older adult falls, sexual violence, and youth sports concussion as important growth areas. In 2015, the Injury Center updated its research priorities in these areas to help guide intramural and extramural investments to accelerate impact at the population level over the next 3-5 years.

CDC funds 10 Injury Control Research Centers (ICRCs) at universities and medical centres to form a national network of academic, public health, and community partners to conduct prevention research, train the next generation of injury prevention researchers and practitioners, and develop and evaluate violence and injury interventions.

Violence is a serious public health problem, affecting people in all stages of life. The Injury Center focuses on primary prevention, investing in surveillance, state and local programmes, and research. In terms of surveillance, CDC's National Violent Death Reporting System (NVDRS) provides states with a clearer understanding of violent deaths to guide local decisions about prevention programmes and track progress. NVDRS is the only state-based surveillance (reporting) system that pools data on violent deaths from multiple sources into a usable, anonymous database.

Programme and research combine in CDC's National Centers of Excellence on Youth Violence Prevention, which prevent youth violence by conducting research, using effective violence surveillance tools, evaluating promising violence prevention programmes, promoting the use of evidence-based and evidence-informed interventions, and strengthening responses to youth violence through community mobilisation efforts.

CDC also provides the best available guidance to the field. Earlier this year, the Injury Center released the STOP-SV technical package, a resource guide for communities to help

Correspondence to Dr Debra Houry, National Center for Injury Prevention and Control, Centers for Disease Control and Prevention, Atlanta, Georgia, 30341 USA; vjz7@cdc.gov.

Competing interests None declared.

Provenance and peer review Commissioned; internally peer reviewed. 
them make decisions about implementing sexual violence prevention strategies. It also released guidance to align efforts to prevent child abuse and neglect in coordination with its Essentials for Childhood framework. These resources identify proven and promising strategies that communities can implement to prevent different types of violence.

Unintentional injuries are the leading cause of death among children and young adults. CDC's Injury Center works with partners to conduct research, evaluate and promote lifesaving strategies that prevent older adult falls, MVCs, opioid overdose and concussions; or reduce their severity when injuries occur.

CDC works with clinical partners to address many issues, including the prevention of older adult falls. The Injury Center developed the Stop Elderly Accidents, Deaths, and Injuries toolkit to help clinicians identify and reduce older adults' risk for falls. The toolkit is based on established clinical guidelines for assessment of fall risk.

The Injury Center also plays a key role by collecting and analysing health-related and MVC--related data, identifies and evaluates interventions to reduce crash risk, and collaborates with partners to provide guidance, resources and tools to improve motor vehicle safety. To assist states with prioritising the implementation of various strategies, CDC has developed an interactive calculator called Motor Vehicle Prioritizing Interventions and Cost Calculator (PICCS). This tool calculates the cost for implementing 14 evidence-based interventions that allow states to factor cost and available resources into their decisions.

CDC also applies its scientific expertise to help curb prescription drug and emerging heroin overdoses. Two critical efforts currently underway are the CDC Guideline for Prescribing Opioids for Chronic Pain (Guideline) and the Prevention for States programme. The Guideline provides 12 recommendations for primary care clinicians about appropriate prescribing of opioids in the treatment of adult patients for chronic pain in the outpatient setting. Prevention for States provides state health departments with resources and support for promoting programmes to reduce prescription drug overdose.

CDC frequently works to fill gaps in the field. For example, the Injury Center is working to improve traumatic brain injury (TBI) surveillance, particularly of sports-related concussions among youth aged 5-21 to address recommendations made within the National Academy of Medicine report on the issue. This system will produce a more complete picture of the incidence and circumstances of brain injuries among Americans, capturing TBIs that are missed currently.

For more than two decades, CDC's Injury Center has sought to reduce injuries and their consequences. Collaborations with students, new professionals and researchers are critical to evaluating current and identifying future prevention strategies to influence trends and protect Americans from injuries and violence. 